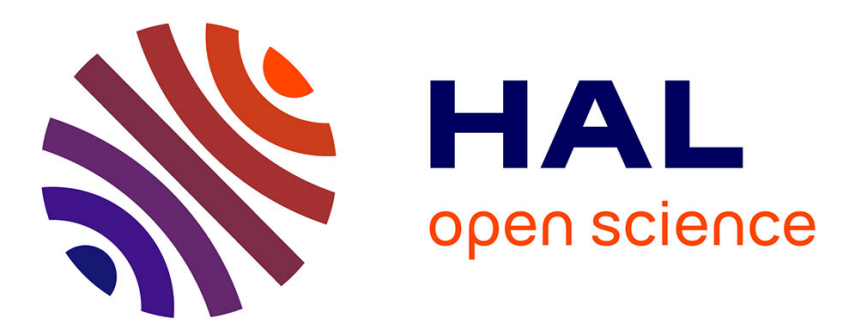

\title{
Etude expérimentale des fluctuations de courant dans une diode à double base au voisinage de son point critique
}

\author{
J. Brini
}

\section{- To cite this version:}

J. Brini. Etude expérimentale des fluctuations de courant dans une diode à double base au voisinage de son point critique. Revue de Physique Appliquée, 1981, 16 (9), pp.517-522. 10.1051/rphysap:01981001609051700 . jpa-00244944

\section{HAL Id: jpa-00244944 https://hal.science/jpa-00244944}

Submitted on 1 Jan 1981

HAL is a multi-disciplinary open access archive for the deposit and dissemination of scientific research documents, whether they are published or not. The documents may come from teaching and research institutions in France or abroad, or from public or private research centers.
L'archive ouverte pluridisciplinaire HAL, est destinée au dépôt et à la diffusion de documents scientifiques de niveau recherche, publiés ou non, émanant des établissements d'enseignement et de recherche français ou étrangers, des laboratoires publics ou privés. 


\title{
Etude expérimentale des fluctuations de courant dans une diode à double base au voisinage de son point critique
}

\author{
J. Brini \\ Laboratoire de physique des composants à semiconducteurs (*), 23, rue des Martyrs, 38031 Grenoble Cedex, France
}

(Reçu le 27 février 1981, révisé le 12 juin 1981, accepté le 16 juin 1981)

\begin{abstract}
Résumé. - Les fluctuations au voisinage du point critique du transistor unijonction sont étudiées systématiquement selon deux approches : à courant constant et à tension interbase constante. Les spectres permettent de différencier deux phénomènes : les fluctuations précédant l'apparition d'oscillations de relaxation, et les fluctuations liées au transport des porteurs dans le semiconducteur. Le rôle limitant de la longueur du barreau dans le ralentissement critique est mis en évidence. On donne également une estimation des exposants critiques concernant la variance et la durée de vie des fluctuations.
\end{abstract}

\begin{abstract}
Fluctuations in the neighbourhood of the critical point of a double base diode are systematically investigated using two types of approach : constant current and constant interbase voltage. Spectra allow the separation of two phenomena : fluctuations announcing the growth of relaxation oscillations, and fluctuations related with the carriers transport in the semiconductor. The device length is shown to have a limiting role on the critical slowing down. Critical exponents are deduced for the variance and the lifetime of the fluctuations.
\end{abstract}

1. Introduction. - On sait que les points critiques des systèmes éloignés de l'équilibre thermo-dynamique présentent un grand nombre d'analogies avec les points critiques des transitions de phase à l'équilibre [1]. Les notions de paramètre d'ordre, de rupture de symétrie, de ralentissement critique, de fluctuations critiques entre autres se sont révélées pertinentes dans les deux classes de phénomènes [2]. Une catégorie particulière de transitions hors d'équilibre apparaît dans les dispositifs électroniques présentant, pour certaines valeurs d'un paramètre extérieur, une résistance électrique dynamique négative. Ainsi, les thermistances [3], les diodes Gunn [4], les transistors unijonctions [5], sont des dispositifs qui, sous réserve d'être polarisés judicieusement, peuvent présenter le phénomène de ralentissement critique, accompagné d'une augmentation considérable du bruit en basse fréquence.

Le présent article expose les résultats expérimentaux relatifs aux fluctuations pour l'un de ces dispositifs, un transistor unijonction (TUJ), polarisé au voisinage du point critique. On sait [6] que la caractéristique d'émetteur du TUJ (Fig. 1) peut prendre, sous l'action du paramètre extérieur $V_{\mathrm{BB}}$ (tension entre bases), une forme du type diode longue (1 Fig. 1) ou une forme en $\mathrm{S}$ (2 Fig. 1). Nous appelons caractéris-

$\left.{ }^{*}{ }^{*}\right)$ Equipe de recherche associée au C.N.R.S. $n^{\circ} 659$.

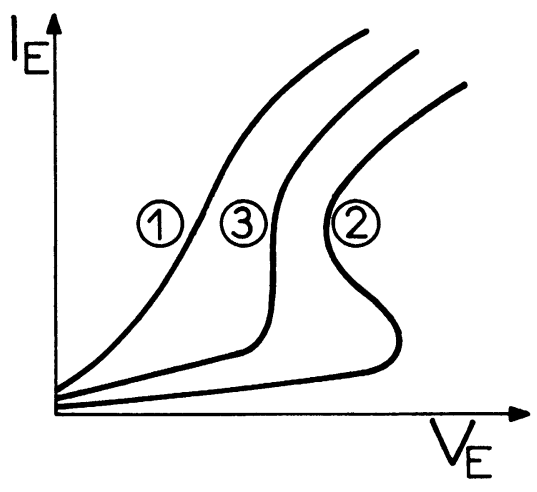

Fig. 1. - Caractéristique d'émetteur d'une diode à double base. [Emitter characteristic of a double base diode.]

tique critique statique la caractéristique intermédiaire (3 Fig. 1), qui comporte une zone importante à résistance dynamique nulle. L'instabilité relative à cette caractéristique est une instabilité en courant, et le problème est analogue à celui de l'arc électrique [7] dual de celui de la diode Gunn.

Nous avons montré par ailleurs [10] que certains exposants critiques relatifs à cette transition prennent des valeurs non classiques lorsqu'on s'approche suffisamment du point critique.

2. Dispositif et conditions de mesure. - Le dispositif étudié est un TUJ industriel du type barreau $(2 \mathrm{~N}$ 
1671) [8]. Il est constitué d'un barreau de silicium légèrement dopé $N$ (dimensions : $2 \times 0,4 \times 0,1 \mathrm{~mm}$ ), au milieu duquel on a réalisé une jonction $p n$ de faibles dimensions. Les porteurs injectés sont par conséquent des porteurs $p$, et l'effet de modulation de conductivité est dû à l'action du champ électrique sur la répartition de ces porteurs dans le barreau.

La figure 2 donne le schéma électrique du montage d'étude.

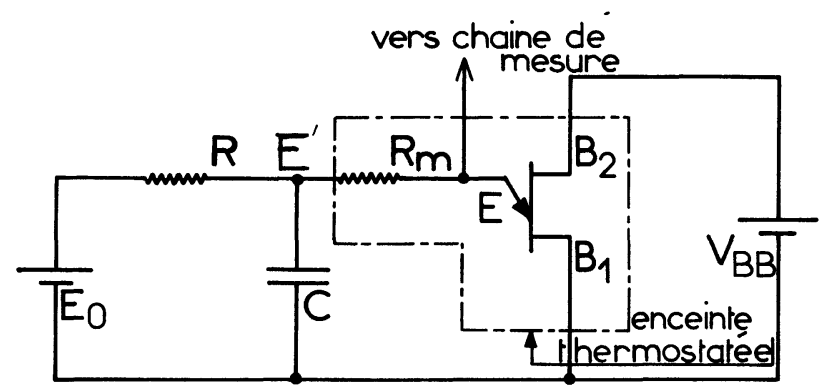

Fig. 2. - Schéma du montage d'étude.

[Diagram of the investigated circuit.]

La tension entre bases $V_{\mathrm{BB}}$ et la tension $E_{0}$ proviennent d'accumulateurs à faible bruit. La résistance $R_{\mathrm{m}}$ est destinée à mesurer le signal de bruit sur l'émetteur $\mathrm{E}$. La capacité $C$, de valeur élevée $(15000 \mu \mathrm{F})$, a pour rôle de mettre le point $\mathrm{E}^{\prime}$ dynamiquement à la masse (court-circuitant ainsi toute fluctuation d'origine extérieure au TUJ). La tension $V_{\mathrm{BB}}$ est ajustée de façon que l'ensemble TUJ $+R_{\mathrm{m}}$ présente une caractéristique $I_{\mathrm{E}}\left(V_{\mathrm{E}^{\prime}}\right)$ critique. Le courant critique est alors, pour le dispositif étudié, $I_{\mathrm{EC}}=95 \mu \mathrm{A}\left({ }^{1}\right)$. L'ensemble TUJ $+R_{\mathrm{m}}$ est plongé dans un thermostat à grande inertie thermique $\left(T=30^{\circ} \mathrm{C} \pm 0,01{ }^{\circ} \mathrm{C}\right)$. Le signal au point $\mathrm{E}$ est étudié à l'aide d'une chaîne numérique d'acquisition et de mesure bâtie autour d'un calculateur T 1600 [9].

La figure 3 donne, dans le plan $I_{\mathrm{E}}\left(V_{\mathrm{E}}\right)$, les points où ont été réalisées les acquisitions. Le point le plus proche du point critique est le point 5 . Nous avons procédé à deux types d'approche de ce point. Le premier concerne les points A, B, C, D, 5. Le courant est maintenu constant et égal au courant critique, $I_{\mathrm{t}}=95 \mu \mathrm{A}$, et la tension $V_{\mathrm{BB}}$ prend des valeurs de plus en plus proches de $V_{\mathrm{BBC}}$. Le paramètre qui mesure la proximité du point critique est alors

$$
\varepsilon=\frac{V_{\mathrm{BB}}-V_{\mathrm{BBC}}}{V_{\mathrm{BBC}}} .
$$

Le deuxième type d'approche du point critique est effectué à $V_{\mathrm{BB}}=V_{\mathrm{BBC}}$, c'est-à-dire sur la caractéristique critique (points 1 à 8 ). Dans ce cas, c'est le cou-

( $\left.{ }^{1}\right)$ Le principe de la détermination précise de $I_{\mathrm{EC}}$ a été exposé ailleurs [10].

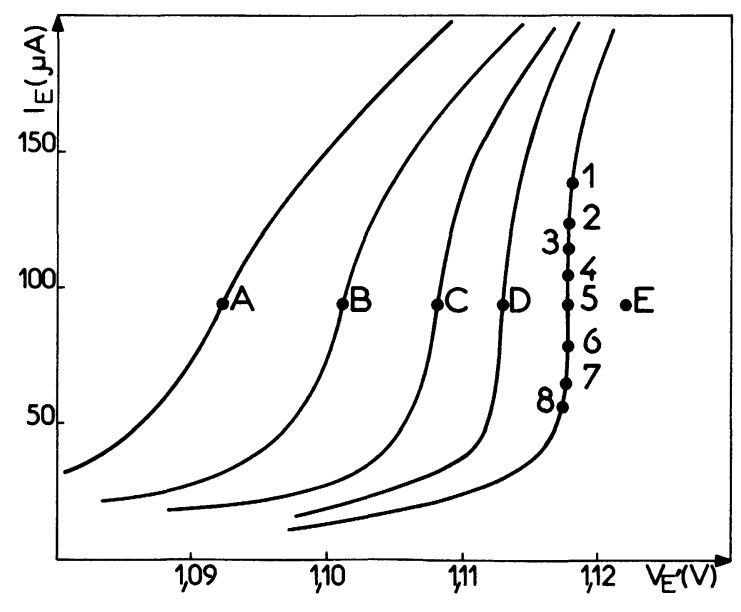

Fig. 3. - Points de mesure dans le plan $I_{\mathrm{E}}\left(V_{\mathrm{E}}^{\prime}\right)$.

[Investigated points in the $I_{\mathrm{E}}\left(V_{\mathrm{E}}^{\prime}\right)$ plane.]

rant qui varie, et la proximité du point critique est mesurée par le paramètre

$$
\Delta=\frac{I_{\mathrm{E}}-I_{\mathrm{EC}}}{I_{\mathrm{EC}}} .
$$

Pour chacun des points mentionnés, nous procédons à 5 acquisitions de 5000 échantillons, à des fréquences d'échantillonnage comprises entre 24 et $12500 \mathrm{~Hz}$. On effectue alors les calculs de l'histogramme d'amplitude, de la variance et de la densité spectrale de puissance (par transformée de Fourier rapide). Les résultats de ces mesures sont exposés ci-après.

3. Histogrammes et variance. - La figure 4 donne un exemple typique des variations d'allure des histogrammes d'amplitude lorsqu'on approche du point critique (points 1 à 5 , fréquence d'échantillonnage de $24 \mathrm{~Hz}$ ). On observe, bien entendu l'élargissement considérable des histogrammes qui correspond aux fluctuations critiques. De plus, on constate que la forme des histogrammes est de plus en plus éloignée d'une gaussienne. L'histogramme E (voir Fig. 3) donne un exemple des formes obtenues légèrement audessus du point critique $\left(V_{\mathrm{BB}}>V_{\mathrm{BBC}}, I_{\mathrm{E}}=I_{\mathrm{EC}}\right)$. On voit que l'émergence des oscillations de relaxation accentue la dissymétrie déjà observable en deçà du point critique. Au simple vu des histogrammes, il n'est donc pas possible de localiser précisément le point critique. On observe seulement une région de dissymétrisation croissante qui exprime le passage graduel d'un signal aléatoire à un signal déterministe. L'éloignement de la forme gaussienne est le témoin d'une forte augmentation de la coopérativité du système, due aux interactions à longue distance entre porteurs dans le barreau. Ce comportement se retrouve quel que soit le mode d'approche du point critique (points A, B, C, D, 5; 1, 2, 3, 4, 5; 8, 7, 6, 5). L'évolution de la variance du signal en fonction des paramètres $\varepsilon$ 


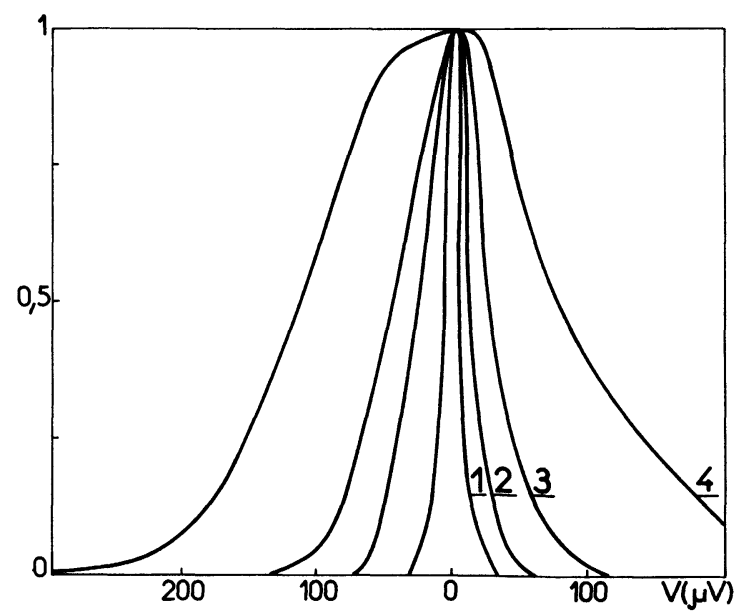

a)

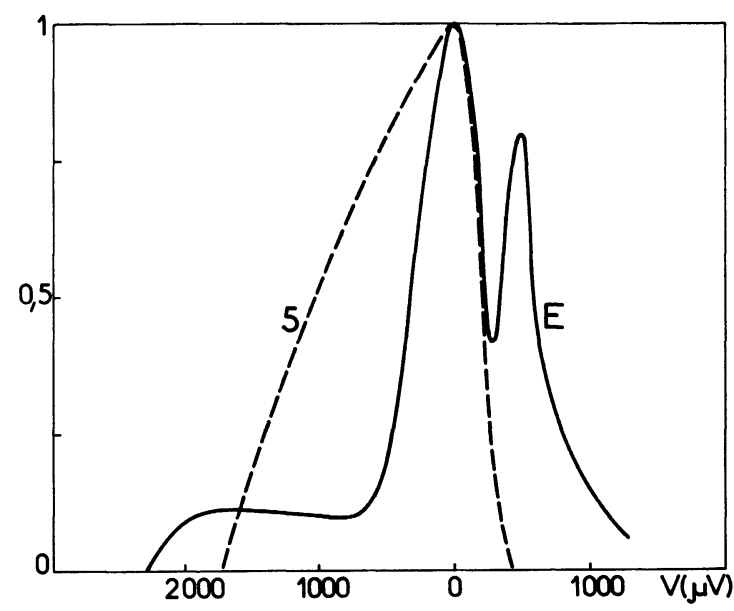

b)

Fig. 4. - a) Histogrammes d'amplitude pour les points 1, 2, 3, 4; b) Histogrammes d'amplitude pour le point critique 5 et le point $\mathrm{E}$.

[a) Amplitude histograms for the points 1, 2, 3, 4; b) Amplitude histograms for the critical point and point $\mathrm{E}$.]

et $\Delta$ est représentée figure $5(a$ et $b)$. La figure $5 a$ montre clairement une variation en $\varepsilon^{-2}$. Cette observation est en désaccord avec l'exposant -1 qui dériverait d'une théorie de champ moyen [11].

L'évolution de la variance en fonction de $\Delta$ est encore plus rapide, et il ne parait pas possible de lui associer un exposant constant. On ne peut que constater une tendance vers un exposant de l'ordre de 2 qui pourrait se manifester par un voisinage plus immédiat du point critique. On peut remarquer en outre que les fluctuations sont plus importantes (à $\Delta$ identique) sur la branche de la caractéristique dont la courbure $\gamma$ est la plus faible. Cette observation permet de prévoir une relation de fluctuation-dissipation faisant intervenir ce paramètre, sans qu'il soit cependant possible de la préciser.

De même que sur les histogrammes il n'est pas possible de distinguer nettement la limite entre signal aléatoire et signal déterministe. Au-delà du point critique, la variance que l'on pourrait mesurer ne

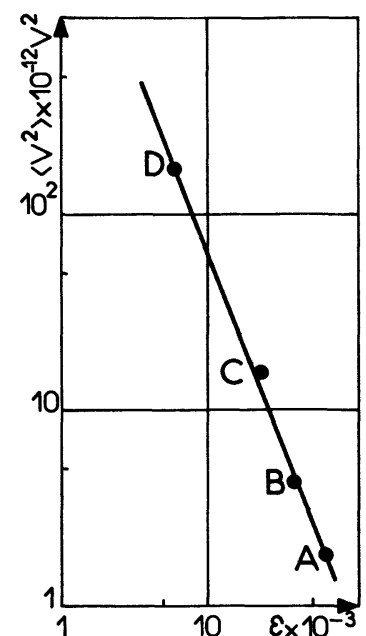

a)

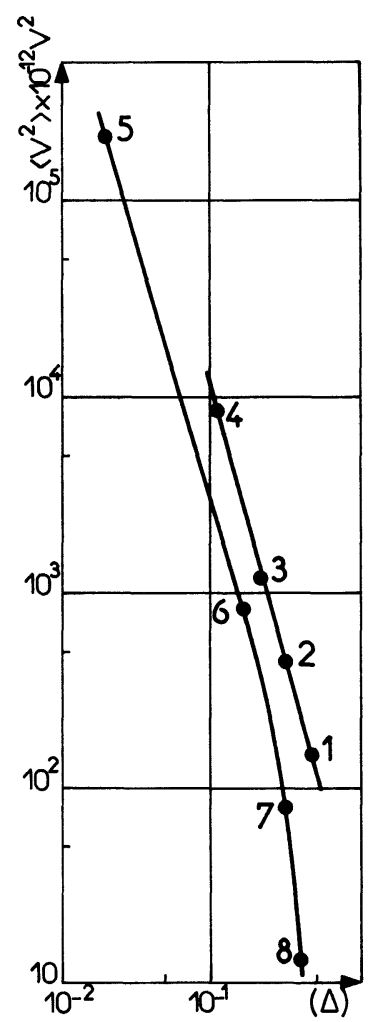

b)

Fig. 5. - Variance en fonction de $\Delta$ et $\varepsilon$.

[Variance versus $\Delta$ and $\varepsilon$.]

ferait que traduire l'augmentation de l'amplitude des oscillations de relaxation qui apparaissent.

4. Densité spectrale de puissance. - Les spectres obtenus pour les points étudiés sont représentés figures $6 a, b, c$. Dans tous les cas, on observe le même type d'évolution.

- Loin du point critique, la densité spectrale de puissance est en $1 / f$ et traduit le bruit en excès imputé selon Mr. Whorter aux effets de recombinaison en 


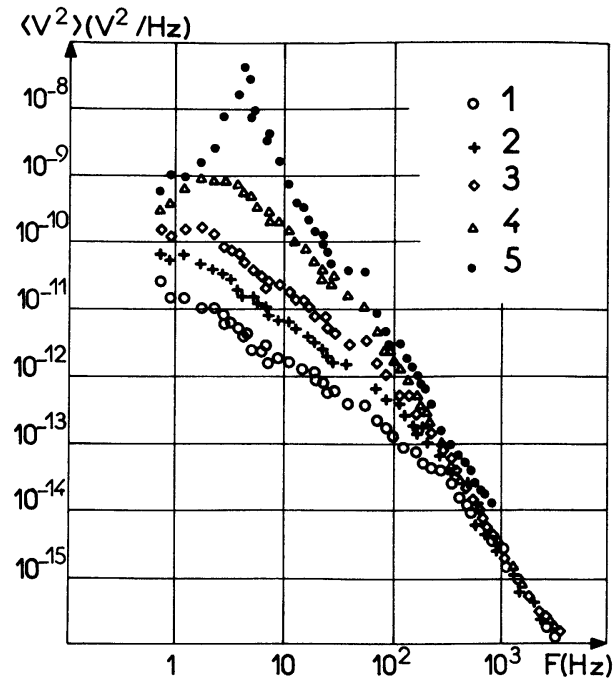

a)

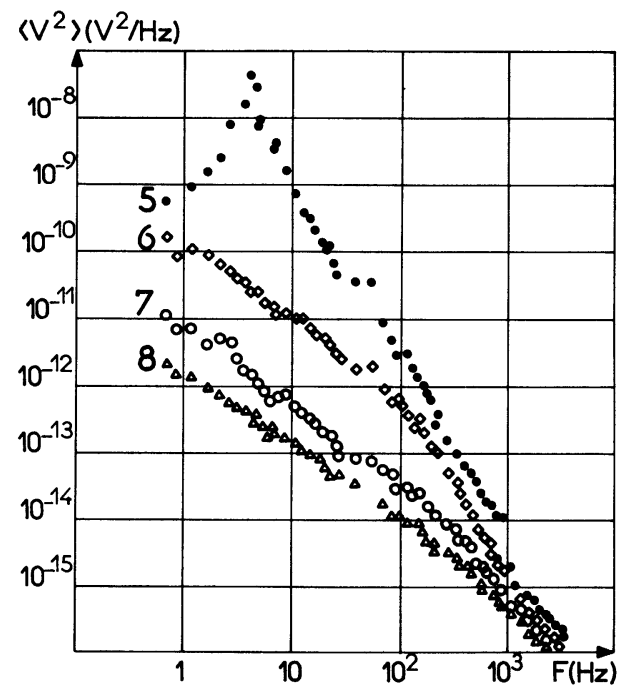

b)

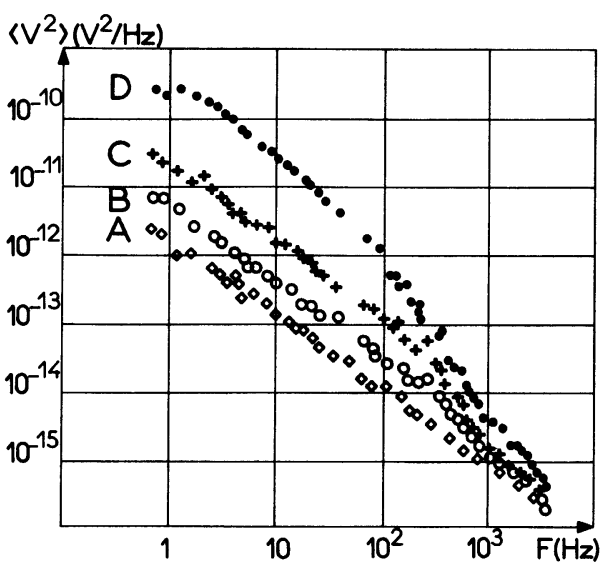

c)

Fig. 6. - Densité spectrale de puissance : $c$ ) en fonction de $\varepsilon$; $a$ ) en fonction de $\Delta>0 ; b$ ) en fonction de $\Delta<0$.

[Power spectral density : c). versus $\varepsilon, a$ ) versus $\Delta>0, b$ ) versus $\Delta<0$.] surface du semiconducteur [12]. La durée de vie des porteurs est trop faible [5,6] pour qu'on puisse observer la rupture de pente associée au bruit générationrecombinaison en volume.

- Lorsqu'on approche du point critique, on observe une rupture de pente entre un spectre en $1 / f$ et un spectre en $1 / f^{2}$. Cette rupture de pente a lieu pour une fréquence $f_{\mathrm{c}}$ d'autant plus basse que l'on est près du point critique, et ceci quel que soit le paramètre de contrôle ( $\varepsilon$ ou $\Delta)$. On peut admettre que la constante de temps $\tau=1 / 2 \pi f_{\text {c }}$ représente la durée de vie des fluctuations dans le volume du barreau. Cette durée de vie est liée à l'ensemble des phénomènes de transport (diffusion, conduction, recombinaison) dans le barreau, et doit être radicalement distinguée de la durée de vie des porteurs.

Corrélativement à l'augmentation de $\tau$, on observe une augmentation globale des fluctuations, nettement plus accentuée pour les basses fréquences.

- Au voisinage immédiat du point critique, la rupture de pente disparaît, simultanément avec l'émergence d'une fréquence privilégiée qui préfigure la fréquence des oscillations de relaxation apparaissant au-delà du point critique.

Le schéma équivalent de bruit du TUJ vu de l'émetteur et du montage est donné figure 7 (d'après [13]). On voit que ce schéma explique bien le pic correspondant à l'apparition des oscillations. Par contre,

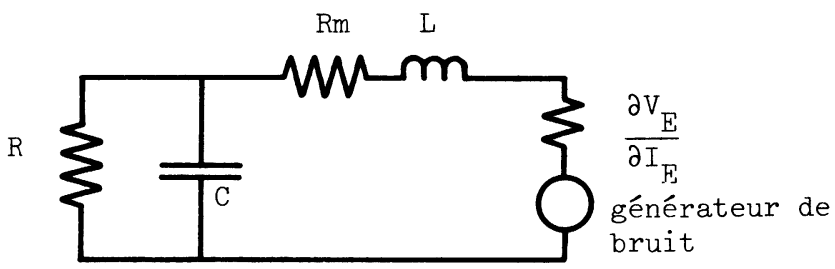

Fig. 7. - Schéma équivalent de bruit du montage d'étude.

[Noise equivalent circuit of the TUJ.]

l'existence d'une rupture de pente à basse fréquence pour $\partial V / \partial I \rightarrow-R_{\mathrm{m}}$ ne peut s'interpréter que par une modification du spectre de bruit du générateur alimentant le circuit. Ce spectre n'est plus, au voisinage du point critique, le spectre classique associé au processus de génération recombinaison des porteurs dans le barreau, mais fait intervenir une constante de temps $\tau$ plus grande que la durée de vie des porteurs. Cette constante de temps exprime à notre avis le ralentissement critique lié à une bifurcation spécifique du TUJ. (Probablement l'apparition d'un filament de courant, dual des domaines à fort champ apparaissent dans une diode Gunn.)

Il convient d'insister sur le fait que les deux phénomènes, rupture de pente et bruit en basse fréquence, ont lieu pour des échelles de temps qui restent distinctes jusqu'au voisinage immédiat du point critique. La fréquence des relaxations, lorsque celles-ci deviennent observables, est de l'ordre de $4 \mathrm{~Hz}$, alors que la 
rupture de pente observée la plus basse a lieu pour $70 \mathrm{~Hz}$. On peut donc affirmer que dans les conditions expérimentales choisies (capacité $C$ de grande valeur) le phénomène macroscopique d'apparition des relaxations, et le phénomène microscopique caractérisé par la rupture de pente sont bien découplés $\left({ }^{2}\right)$.

La figure $8 a, b$ montre l'évolution de la constante de temps $\tau=1 / 2 \pi f_{\mathrm{c}}$ avec les paramètres $\Delta$ et $\varepsilon$. Il apparaît que la variation de $\tau$ est différente suivant le paramètre de contrôle choisi. L'évolution de $\tau$ avec $\Delta$ (Fig. $8 b$ ) est compatible avec un exposant critique de l'ordre de $-1\left(\tau \sim \Delta^{-1}\right)$ alors que l'évolution avec $\varepsilon$ est plus rapide.

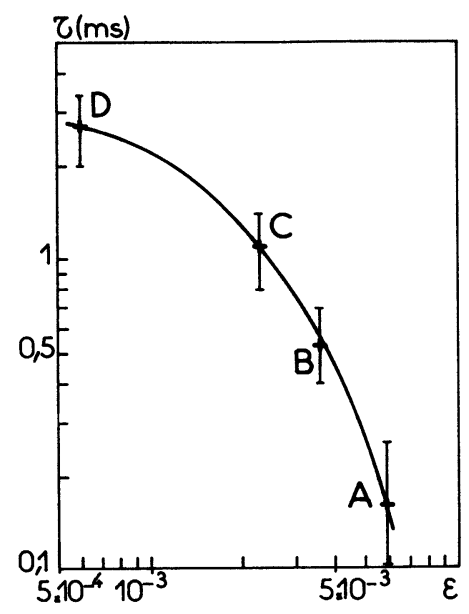

a)

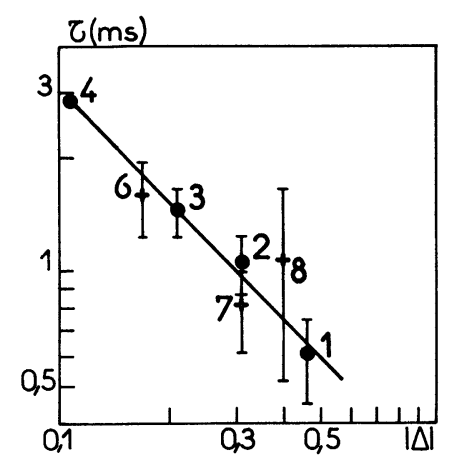

b)

Fig. 8. - Variation de $\tau$ en fonction de $\varepsilon$ et $|\Delta|$.

[Evolution of $\tau$ versus $\varepsilon$ and $\Delta$.]

De plus, on peut constater qu'au-delà d'une valeur maximale de $\tau, \tau_{\max } \# 3 \mathrm{~ms}$, la rupture de pente disparait. Comme l'essentiel de la conduction dans le barreau est assuré par les trous injectés au niveau de l'émetteur, on peut associer à $\tau$, une longueur de diffusion des fluctuations $L=\sqrt{D_{\mathrm{p}} \tau}$, où $D_{\mathrm{p}}$ est le coefficient de diffusion des trous. On constate alors que la

$\left({ }^{2}\right)$ Lorsque la capacité $C$ est plus faible, il existe une zone de paramètre de contrôle où les deux phénomènes se superposent : il y a coalescence sur le spectre de la fréquence correspondant à la rupture de pente et de la fréquence d'amorçage. longueur $L$ associée à $\tau_{\max }$ est de l'ordre de grandeur de la longueur totale $L_{\mathrm{B}}$ du barreau $(2 \mathrm{~mm})$. Cette remarque est importante pour la raison suivante : la répartition des porteurs dans le barreau est régie par l'équation de transport ambipolaire [18] :

$$
-\frac{\delta p}{\tau}+\mu^{*} E \frac{\partial \delta p}{\partial x}+D^{*} \partial^{2} \frac{\delta n}{\partial x^{2}}=0
$$

où

$$
\mu^{*}=\mu_{n} \mu_{p} \frac{n-p}{\mu_{p} p+\mu_{n} n} \# \mu_{\mathrm{p}}
$$

est la mobilité ambipolaire, et

$$
D^{*}=D_{n} D_{p} \frac{n+p}{D_{n} n+D_{p} p} \# D_{p}
$$

est le coefficient de diffusion ambipolaire.

A l'équation (1) sont associées les racines de l'équation caractéristique :

$$
r_{1,2}=\frac{\mu^{*} E \tau \pm \sqrt{\mu^{* 2} E^{2} \tau^{2}+4 D^{*} \tau}}{2 D^{*} \tau}
$$

qui se réduisent à $r_{1,2}=1 / \sqrt{D^{*} \tau}$ lorsque le terme $\mu^{* 2} E^{2} \tau^{2}$ devient négligeable devant $4 D^{*} \tau$. Si la durée de vie maximale $\tau_{\max }$ associée aux fluctuations critiques est de l'ordre de grandeur de $L_{\mathbf{B}}^{2} / D_{p}$, cela suggère donc que, dans l'équation de transport des fluctuations, le phénomène de conduction est négligeable devant le phénomène de diffusion. Nous rejoignons par-là une hypothèse avancée par Berlan [5] à partir de considérations purement macroscopiques.

De plus, nous montrons ici que la longueur du barreau joue un rôle limitant pour le ralentissement critique. Cette hypothèse demande bien entendu à être vérifiée par l'étude de TUJ de longueurs différentes.

5. Commentaire. - On rapproche généralement le ralentissement observé au voisinage d'une transition hors d'équilibre du ralentissement critique associé aux transitions de phase classiques. Il semble cependant que dans notre cas ce rapprochement ne soit pas totalement pertinent. En effet, les spectres obtenus mettent en évidence deux phénomènes simultanés qui peuvent tous deux être désignés par le terme de ralentissement critique.

Le premier est l'augmentation des fluctuations en basse fréquence qui précède l'apparition des oscillations de relaxation. Ce phénomène a été observé pour de nombreux oscillateurs, comme l'oscillateur à pont de Wien [14], l'oscillateur de Van der Pol [15], l'oscillateur à diode Gunn [16], le laser Monomode [17]. Il est lié à une bifurcation foyer stable $\rightarrow$ foyer instable + cycle limite pour le système macroscopique $\left({ }^{3}\right)$.

$\left({ }^{3}\right)$ Des mesures de la variance et du temps de corrélation au voisinage de ces bifurcations ont permis à Kawakuko et al. de mettre en évidence des exposants critiques compatibles avec les théories de champ moyen [15]. 
Le deuxième phénomène est l'augmentation de la constante de temps propre aux phénomènes de transport dans le barreau. Cette augmentation est liée à une bifurcation dont la nature reste à déterminer à l'aide des équations de transport des porteurs dans le barreau [13]. La dualité avec la diode Gunn laisse cependant prévoir que cette bifurcation pourrait correspondre à l'apparition de domaines à fort courant (filaments de courant). Son identification nécessiterait par conséquent une résolution des équations de transport complètes à 2 dimensions.

Les deux phénomènes apparaissent simultanément parce que le second (formation de domaines) provoque le premier (naissance des oscillations) par le biais de l'apparition d'une résistance négative. Du point de vue des fluctuations, cependant, il convient de les distinguer nettement, l'un étant la source de bruit qui perturbe l'autre : l'augmentation de la durée de vie des fluctuations dans le barreau entraîne une modification du spectre des fluctuations de la conductance du barreau qui intervient dans les fluctuations de l'oscillateur. De ce fait, le fonctionnement de ce dernier ne peut en aucun cas être traité à l'aide d'un formalisme de Langevin. En toute rigueur, l'oscillateur doit être décrit par un système non linéaire dont les coefficients sont des fonctions aléatoires elles-mêmes dépendantes des paramètres de contrôle. Un tel problème soulève malheureusement des difficultés mathématiques insurmontables [19]. On conçoit cependant que le système puisse présenter des écarts notables avec les théories de champ moyen.

6. Conclusion. - L'étude systématique des fluctuations au voisinage du point critique du TUJ met en évidence le ralentissement critique spécifique lié au transport des porteurs dans le barreau. Ce ralentissement est distinct des fluctuations liées à l'apparition d'oscillations macroscopiques. Il est caractérisé par une augmentation de la durée de vie des fluctuations visible sur le spectre grâce au déplacement de la rupture de pente entre un spectre en $1 / f$ et un spectre $1 / f^{2}$. Les dimensions géométriques du barreau semblent jouer un rôle limitant pour cette augmentation.

Notre étude permet également une estimation des exposants critiques pour la variance des fluctuations et leur durée de vie, en fonction des deux paramètres de contrôle $\varepsilon$ et $\Delta$. On obtient pour la variance une évolution en $\varepsilon^{-a}$ avec $a \sim 2$ et en $\Delta^{-b}$ avec $b \geqslant 2$. Pour la durée de vie des fluctuations, la variation est en $\varepsilon^{-1}$ et en $\Delta^{-1}$.

Remerciements. - Ce travail n'aurait pu voir le jour sans les fructueuses discussions que nous avons eues avec Monsieur Papoular, Maitre de Recherche au CNRS.

\section{Bibliographie}

[1] HaKen, H., Synergetics, Teubner, Stuttgart (1973).

[2] Haken, H., Rev. Mod. Phys. 47 (1975) 67.

[3] Landauer, R., Phys. Rev. A 15 (1977) 2117.

[4] Nakamura, K., J. Phys. Soc. Jpn 38 (1975) 46.

[5] Berlan, D., Thèse INPG, Grenoble (1977).

[6] Berlan, D., Viktorovitch, P., Kamarinos, G., Physica 92 B + C (1977) 14.

[7] Nozières, P., Phénomènes critiques, cours organisés par la SFP, Grenoble (1978-1979).

[8] Clark, L., Electronics June (1965) p. 93.

[9] Rahal, S., Thèse INPG, Grenoble (1980).

[10] Brini, J., C.R. Hebd. Séan. Acad. Sci. Paris, série B 290 (1980) 5 .
[11] Stanley, H. E., Oxford, Clarendon Press (1971).

[12] MrWhorTer, M. I. T., Lincoln Lab. Rep. no 80 (1955).

[13] Scharfeter, D. L., Jordan, A. G., IRE Trans. ED9 (1962) p. 461.

[14] Kawakubo, T., Kabashima, S., J. Phys. Soc. Jpn 37 (1974) 1199.

[15] Kawakubo, T., Kabashima, S., Ojishima, M., J. Phys. Soc. Jpn 34 (1973) 1169.

[16] Kawakubo, T., Kabashima, S., Nishimura, K., J. Phys. Soc. Jpn 34 (1973) 1460.

[17] Pytte, E., Thomas, H., Phys. Rev. 179 (1969) 431.

[18] Kamarinos, G., Thèse INPG, Grenoble (1974).

[19] Crandall, S. H., J. Acoust. Soc. Am. 35 (1963) 1700. 